\title{
Implementation of the Tobacco Tactics intervention versus usual care in Trinity Health community hospitals
}

Sonia A. Duffy ${ }^{1,2^{*}}$, David L. Ronis ${ }^{3}$, Lee A. Ewing ${ }^{4}$, Andrea H. Waltje ${ }^{5}$, Stephanie V. Hall ${ }^{4}$, Patricia L. Thomas ${ }^{6}$, Christine M. Olree ${ }^{7}$, Kimberly A. Maguire ${ }^{8}$, Lisa Friedman ${ }^{9}$, Sue Klotz ${ }^{10}$, Neil Jordan ${ }^{11,12}$ and Gay L. Landstrom ${ }^{13}$

\begin{abstract}
Background: Guided by the Reach, Effectiveness, Adoption, Implementation, and Maintenance (RE-AIM) implementation framework, a National Institutes of Health-sponsored study compared the nurse-administered Tobacco Tactics intervention to usual care. A prior paper describes the effectiveness of the Tobacco Tactics intervention. This subsequent paper provides data describing the remaining constructs of the RE-AIM framework.

Methods: This pragmatic study used a mixed methods, quasi-experimental design in five Michigan community hospitals of which three received the nurse-administered Tobacco Tactics intervention and two received usual care. Nurses and patients were surveyed pre- and post-intervention.

Measures included reach (patient participation rates, characteristics, and receipt of services), adoption (nurse participation rates and characteristics), implementation (pre-to post-training changes in nurses' attitudes, delivery of services, barriers to implementation, opinions about training, documentation of services, and numbers of volunteer follow-up phone calls), and maintenance (continuation of the intervention once the study ended).
\end{abstract}

Results: Reach: Patient participation rates were $71.5 \%$. Compared to no change in the control sites, there were significant pre- to post-intervention increases in self-reported receipt of print materials in the intervention hospitals $(n=1370, p<0$. 001). Adoption: In the intervention hospitals, all targeted units and several non-targeted units participated; 76. $0 \%(n=1028)$ of targeted nurses and 317 additional staff participated in the training, and $92.4 \%$ were extremely or somewhat satisfied with the training. Implementation: Nurses in the intervention hospitals reported increases in providing advice to quit, counseling, medications, handouts, and DVD (all $p<0.05$ ) and reported decreased barriers to implementing smoking cessation services $(p<0.001)$. Qualitative comments were very positive ("user friendly," "streamlined," or "saves time"), although problems with showing patients the DVD and charting in the electronic medical record were noted. Maintenance: Nurses continued to provide the intervention after the study ended.

Conclusions: Given that nurses represent the largest group of front-line providers, this intervention, which meets Joint Commission guidelines for treating inpatient smokers, has the potential to have a wide reach and to decrease smoking, morbidity, and mortality among inpatient smokers. As we move toward more population-based interventions, the RE-AIM framework is a valuable guide for implementation.

Trial registration: ClinicalTrials.gov, NCT01309217

Keywords: Smoking, Cessation, Inpatient

\footnotetext{
* Correspondence: Duffy.278@osu.edu

${ }^{1}$ College of Nursing, Ohio State University, Newton Hall, 1585 Neil Ave,

Columbus, OH 43210, USA

${ }^{2}$ Veterans Affairs (VA) Center for Clinical Management Research, HSR\&D

Center of Excellence, 2215 Fuller Road, Ann Arbor, MI 48105, USA

Full list of author information is available at the end of the article
}

(c) The Author(s). 2016 Open Access This article is distributed under the terms of the Creative Commons Attribution 4.0 International License (http://creativecommons.org/licenses/by/4.0/), which permits unrestricted use, distribution, and reproduction in any medium, provided you give appropriate credit to the original author(s) and the source, provide a link to the Creative Commons license, and indicate if changes were made. The Creative Commons Public Domain Dedication waiver (http://creativecommons.org/publicdomain/zero/1.0/) applies to the data made available in this article, unless otherwise stated. 


\section{Background}

Smoking cessation interventions that include counseling, medications, and telephone follow-up for hospitalized smokers have been shown to be efficacious [1]. Hospitalization provides an excellent opportunity for patients to quit smoking because they are a captive audience, are often motivated to quit due to illness, and often quit temporarily due to hospital smoking bans. Moreover, meta-analyses suggest that nurse-administered interventions are efficacious, particularly among hospitalized patients [2]. Despite the strong evidence for the efficacy of inpatient smoking interventions, a large gap exists between the availability of effective smoking cessation interventions and their widespread dissemination in hospitals [3-5]. The challenge rests with disseminating smoking cessation interventions into standard practice.

The Reach, Effectiveness, Adoption, Implementation, and Maintenance (RE-AIM) framework has been used in other dissemination and implementation health behavior and smoking studies [6-15]. Utilization of the RE-AIM framework is intended to enhance the applicability of research-based interventions in clinical practice and ease the process of planning, conducting, reporting, and selecting interventions to be implemented on a large scale [16]. The constructs of the RE-AIM framework are the following: (1) reach (percent and representativeness of individuals receiving an intervention); (2) effectiveness (impact of an intervention on outcomes); (3) adoption (proportion and representativeness of settings and providers willing to deliver the intervention); (4) implementation (extent to which the intervention is implemented as intended); and (5) maintenance (sustainability of an intervention at individual and setting levels) [17]. The RE-AIM framework has been used to guide several studies testing the Tobacco Tactics intervention.

A randomized controlled trial $(n=184)$ tested the efficacy of the Tobacco Tactics intervention among head and neck cancer patients and found that 6-month smoking cessation rates were $47 \%$ quitting in the Tobacco Tactics group compared to $31 \%$ in the usual care group $(p<0.05)$. Ninety percent of participants said they would recommend the intervention and the manual to someone else dealing with similar issues. As with many efficacy studies, the intervention was not maintained and ended when the trial ended [18].

The Tobacco Tactics intervention was then packaged into a toolkit for inpatient nurses and smokers in the Department of Veterans Affairs (VA) [19]. Reach: Compared to the usual care site, patients in the intervention sites reported an increase in receiving and satisfaction with selected cessation services, particularly medications $(p<0.05)$ [19]. Effectiveness: Six-month quit rates improved from the pre- to the post-intervention time periods in Ann Arbor $(p=0.004)$ and Detroit $(p<0.001)$ (which services a large number of African Americans), compared to the Indianapolis control site $(n=1070)$. Adoption: Three hundred sixty-nine or $74 \%$ of targeted nurses and 282 non-targeted personnel were trained in the Tobacco Tactics intervention. Implementation: Nurses' self-reported administration of cessation services increased from $57 \%$ pre- to $86 \%$ post-training $(p=0.0002)$. The intervention was incorporated into new nurse training, and maintenance was high as the programs remain in place in Ann Arbor and Detroit 3 years after the study ended [20]. The intervention was exported to another VA via satellite broadcast where it has continued to be implemented 2 years after the study ended [21].

A recently completed NIH-supported R21 randomized controlled trial compared a nurse-supported, web-based version of the Tobacco Tactics intervention versus referral to the 1-800-QUIT-NOW telephone quitline $(N=145)$ among blue collar workers. Efficacy: The Tobacco Tactics website group showed significantly higher quit rates $(n=18 / 67,26.9 \%)$ than the 1-800-QUIT-NOW group $(n=6 / 78, \quad 7.7 \%)$ at 1-month follow-up $(p=0.003)$. Reach: Compared to participants in the 1800-QUIT-NOW group, significantly more of those in the Tobacco Tactics website group participated in the intervention, received phone calls and nicotine replacement therapy (NRT), and found the intervention helpful [22].

These prior studies show that the Tobacco Tactics intervention had high reach, efficacy/effectiveness, adoption, implementation, and maintenance in VA, non-VA, and community settings. As one of these six studies funded by the National Institutes of Health, which together comprised the Consortium of Hospitals to Advance Research on Tobacco (CHART), this pragmatic trial used the RE-AIM framework [12, 13, 23, 24] to test the nurse-administered Tobacco Tactics intervention on inpatient units in six community hospitals. This study differed from the other CHART trials in that (1) this was a non-randomized, implementation trial while the others were all randomized controlled studies and (2) all but one of the other trials tested variations on referral to quit telephone lines, the other tested referral to website, while this study used real-world staff nurses to deliver the intervention [25-33].

A recently published paper in the American Journal of Preventive Medicine describes the effectiveness of the Tobacco Tactics CHART study [34]. There were significant improvements in propensity-adjusted, pre- to postintervention self-reported quit rates and cotinine-verified quit rates in the intervention sites compared to no change in the control sites. Excluding the recently published effectiveness results, the specific aims of this study were to

(1)determine the reach of the intervention by identifying patient participation rates, characteristics 
of the patient sample, and number of patients that self-reported receiving the intervention;

(2) determine the adoption of the intervention by describing the units that participated in the study, nurse survey participation rates, characteristics of the nurses, number of nurses trained, and nurses' opinions about the training;

(3) determine the implementation of the intervention by describing changes in nurses' attitudes toward providing cessation services, delivery of the components of the intervention, barriers to implementation, documentation of services, and number of volunteer follow-up phone calls made; and

(4) determine the maintenance of the intervention by evaluating short-term sustainability of the intervention once the study ends.

\section{Methods}

\section{Design}

Details of the study design have been described in a published protocol paper [25]. Using data collected from the larger study, this paper provides information on the process evaluation using the RE-AIM framework, excluding effectiveness (outcome) evaluation, which was recently published separately [34]. Using mixed methods, this quasiexperimental study initially designed to be conducted among a convenience sample of six Michigan Trinity Health community hospitals (matched on size and number of minority patients), of which three were to receive the nurse-administered Tobacco Tactics intervention and three were to receive usual care, although data from one of the control hospitals was not useable due to a protocol deviation. Not to randomize, but only to reduce investigator bias, a random number generator was used to assign the hospitals to experimental and control conditions. While medical surgical units were the primary units targeted, the leaders at the hospitals were allowed to include additional units, which increased the generalizability of study findings. However, nurses and patients on nontargeted units were not followed [25]. Human study approval was received from the University of Michigan (Health Sciences and Behavioral Sciences Institutional Review Board \#HUM00043349) and Trinity Health hospitals (Mercy Health Institutional Review Board \#2011013, Saint Joseph Mercy Institutional Review Board \#HSR-11-1272, and Saint Mary's Health Care Institutional Review board \#SM11-830-01).

\section{Setting and sample \\ Setting}

Trinity Health is one of the largest multi-institutional Catholic health care delivery systems in the nation. Committed to those who are poor and underserved in its communities, Trinity Health serves people and communities in 21 states with 124 continuing care locations, and 91 hospitals, of which five Michigan hospitals were included in the study.

\section{Patient sample}

Inclusion criteria for the study were patients that (1) smoked a cigarette within 1 month prior to hospitalization, (2) were at least 18 years of age, and (3) had a projected hospital stay of at least $24 \mathrm{~h}$. Excluded were smokers that were (1) involved in a concurrent smoking cessation trial, (2) non-English speaking, or (3) not cognitively or physically able to participate.

\section{Nurse sample}

Dissemination/implementation research requires attention not only to the individual, but also to the staff and organization delivering the intervention [35]. The nurse sample included nurses from participating inpatient units.

\section{Procedures \\ Reach and effectiveness}

To obtain population quit rates (presented in a prior paper [34]) and patient feedback on tobacco services received, throughout the entire study, all inpatient smokers were identified from the electronic medical record (EMR) and approached by a research assistant to provide written informed consent and a survey. Using a modified Dillman approach, patients were mailed surveys 30 days and 6 months after discharge [36]. Participants were given $\$ 10$ for each survey returned. At 6 months post-discharge, participants that returned 6-month surveys were provided a urinary NicAlert cotinine test strip to be mailed back, for which they received an additional \$20 [37]. Medical information, receipt of cessation services, and quit rates for those lost to follow-up were also downloaded or abstracted from the EMR. Midway through the study, nurses in the intervention hospitals were trained in the Tobacco Tactics intervention, which became the standard of care for treating tobacco dependence for all inpatient smokers in the institution, whether or not they enrolled in the study. In this way, receipt of services and quit rates for all patients were determined pre-intervention, during training (transition period), and post-intervention in both intervention and control groups. Data from patients in the transition period, while nurses were being trained, were not included in the analyses.

\section{Adoption, implementation, and maintenance}

Nurses in both intervention and control hospitals were anonymously surveyed pre-intervention to assess attitudes about, provision of, and barriers to providing smoking cessation services to inpatient smokers. In the intervention hospitals only, working with the research nurse, master trainers then provided 1-h training 
sessions on all shifts to targeted nurses who were then immediately surveyed about their opinions about the training. Approximately 3 months after training, nurses in intervention and control hospitals were again surveyed to assess attitudes about, provision of, and barriers to providing smoking cessation services to inpatient smokers. In addition, $10 \%$ of targeted nurses in the intervention sites were interviewed. The training was incorporated into orientation for all new nurses.

\section{Description of the Tobacco Tactics intervention Tobacco Tactics toolkit for nurses}

Based on Agency for Healthcare Research and Quality guidelines, the cessation toolkit included (1) one continuing education unit (CEU) contact hour for training, (2) a PowerPoint presentation on behavioral and pharmaceutical interventions, (3) a pocket card "Helping Smokers Quit: A Guide for Clinicians" developed by the US Department of Health and Human Services, Public Health Service, (4) behavioral and pharmaceutical protocols, and (5) a computerized template for nurse documentation based on the components of Joint Commission (JC) Smoking Cessation standards [38].

\section{Tobacco Tactics toolkit for patients}

For patients, the cessation toolkit included (1) a brochure, (2) a cessation DVD, (3) the Tobacco Tactics manual [39], (4) a 1-800-QUIT-NOW card, (5) nurse behavioral counseling and pharmaceuticals, (6) physician reminder to offer brief advice to quit coupled with medication sign-off, and (7) follow-up phone calls.

\section{Volunteer telephone counseling}

When the nurse charted on the documentation template that the patient was given the Tobacco Tactics manual, the EMR was programmed to add the patient's name and phone number to a list that was forwarded to Voluntary Services two times per week. Trained volunteers at each hospital provided telephone cessation counseling to patients at 2, 7, 14, 21, and 30 days after discharge [20]. Volunteers did not collect research data but did provide documentation that was entered into the EMR.

\section{Description of usual care}

In the Trinity Health System, all inpatients were screened for smoking via the nursing assessment. Nurses were instructed to give smokers brief advice to stop smoking and a brochure.

\section{Measures}

The reach of the intervention was measured by calculating patient participation rates and follow-up rates (number of participating/number patients eligible) from recruitment logs. From patient surveys and chart audits, the characteristics of the patient sample were described including demographic characteristics, discharge diagnosis and discharge comorbidity ICD-9 codes categorized according to standard categories [40], the Patient Health Questionnaire 2 (PHQ-2) for depression [41], the Alcohol Use Disorders Identification Test-C (AUDIT-C) for alcohol use [42, 43], and number of cigarettes smoked per day. Differences in pre- to post-intervention patient self-reported receipt of tobacco services were calculated from patient surveys. The adoption of the intervention was measured by describing the units that participated in the study and nurse participation rates in pre- and post-intervention surveys and trainings (number of nurses participating/number eligible) from recruitment logs. Pre- and post-intervention nurse surveys provided information on the characteristics of the nurses and nurses' opinions about the training (overall satisfaction, satisfaction with pharmaceutical management, satisfaction with behavioral management, understanding of training, and helpfulness of training, all of which were rated on a fivepoint Likert scale).

Implementation was measured from nurse surveys by calculating pre- to post-training changes in self-reported attitudes toward providing cessation services, delivery of the components of the intervention, and barriers to implementation. In the intervention sites only, implementation was measured via descriptive statistics of documentation of services downloaded from the EMR. In the intervention sites only, interviews were conducted with nurses about their provision of Joint Commission measures for inpatient tobacco cessation (yes/no) and delivery of components of the intervention (yes/no), and nurses were asked to give qualitative comments about their experience implementing the intervention. Volunteer logs revealed the number of phone follow-up attempts, percent reached, average number of calls per patient, number of patients reached, and total number of contacts. Maintenance of the intervention was measured by determining whether the implementation continued after the researchers withdrew support from the hospitals as noted in postintervention patient and nurse surveys (short-term maintenance) and anecdotal communication with nurses after the study ended (long-term maintenance). See Additional files 1, 2, and 3 for details on the measures.

\section{Data analysis}

Descriptive statistics were used to summarize all variables. Patient and nurse pre- to post-intervention differences in the intervention and control sites were compared using chi-square tests of association and $t$ tests. The significance level was set at $\alpha=0.05$, and a 
two-tailed test was conducted. All analyses were conducted using SPSS version 21 software.

Qualitative data from the structured nurse interviews was coded by two members of the research team. Disagreements were discussed by the coders and resolved. Themes were based on nurses' experiences implementing specific components of the intervention (e.g., DVD, Tobacco Tactics manual). The qualitative results were triangulated with quantitative results, which involve cross verifying the same information from different sources, in this case comparing patient data, nurse survey and interview data, and EMR data. It was expected that the rich qualitative data obtained from a smaller number of nurse interviews could further explain the more closed-ended, but more generalizable, patient and nurse surveys and EMR data that could be obtained from larger numbers of participants.

\section{Results}

Reach

Patient participation and follow-up rates from recruitment logs, EMR downloads, and patient surveys

Recruitment logs showed that across the three intervention and two control hospitals, 4013 smokers were approached from October 2011 through May 2013. Of these, 2136 were eligible, 608 refused, and 1528 were enrolled ( $71.5 \%$ participation rate) of which 158 were in the transition period while nurses were being trained and were therefore not included in the analysis. The patient follow-up rates for intervention and control sites were similar at $61.5 \%(n=641 / 1042)$ and $61.3 \%$ $(n=298 / 486)$, respectively. Patient surveys and EMR data showed that non-responders to the follow-up survey were more likely to be male $(p<0.001)$, employed $(p<0.01)$, and have a primary diagnosis of mental disorder $(p=0.001)$. See effectiveness paper for recruitment and retention flowchart [34].

\section{Patient characteristics from patient surveys and EMR downloads}

The description of the sample can be seen in Table 1. The average age was 47.9 years old, just over half were females, and three quarters were white race, and just under one third were married. On average, participants smoked about 15.4 cigarettes per day and $10.0 \%$ used other tobacco products. About one third screened positive for probable problem drinking and one third screened positive for probable depression. Just over one quarter had a diagnosis of unipolar disorder and about one in five had a diagnosis of substance use disorder. The most common discharge diagnoses were diseases of the digestive system (13.1\%), diseases of the circulatory system (13.1\%), injury and poisoning (9.9\%), and diseases of the respiratory system $(8.9 \%)$. The most common comorbidities were endocrine, nutritional, and metabolic diseases and immunity disorders (60.2 \%), diseases of the circulatory system (53.3\%), mental disorders (50.4\%), diseases of the digestive system (35.5\%), symptoms, signs, and ill-defined conditions (35.4\%), and diseases of the respiratory system (34.3\%).

\section{Self-reported receipt of tobacco services from patient surveys}

In the intervention sites, more patients $(39.9 \%)$ in the post-intervention period reported receiving handout materials compared to the pre-intervention period $(28.4 \%)(p<$ 0.001 ), whereas there was a decrease in receipt of handout materials in the control group pre- to post-intervention (30.2\% pre- versus $20.5 \%$ post-intervention; $p<0.01$ ).

\section{Adoption}

Description of participating units from recruitment logs and related notes

All targeted medical surgical units and several nontargeted units participated. In one intervention site, obstetrics/gynecology (OB/GYN) nurses requested participation and were given specialized training on tobacco cessation with pregnant smokers and, since there are no evidence-based guidelines for medications with this population, nurses were counseled to work closely with the patient's obstetrician. Labor and delivery nurses also performed the intervention with expectant fathers, although fathers were not technically patients and therefore could not be given medications and were not followed. This same hospital chose to include the cardiac care unit $(\mathrm{CCU})$, progressive care unit (PCU), and medical detoxification unit but not the psychiatric/behavioral health and cardiovascular and thoracic surgery units, yet nurses on these units requested and were provided with materials. Another intervention site chose to include the CCU. A third intervention site chose to train all outpatient nurses. One control site chose to include the behavioral health/substance abuse unit and another site chose to include the acute rehabilitation unit.

\section{Participation in pre-intervention nurse surveys from recruitment logs}

Across both intervention and control hospitals, $76.7 \%$ ( $n=1403 / 1829)$ of targeted nurses and 317 non-targeted providers returned pre-intervention surveys, for a total of 1720 participants. Pre-intervention nurse survey response rates were $76.0 \%(n=1028 / 1352)$ at intervention sites and $78.6 \%(n=375 / 477)$ at control sites.

\section{Characteristics of nurses in pre-intervention surveys}

Characteristics of the pre-intervention nurses showed that $37.0 \%$ were less than 35 years old, $45.9 \%$ were 35 to 54 years old, and $17.1 \%$ were greater than 54 years old. Nearly all were female (92.5\%), non-Hispanic 
Table 1 Baseline characteristics of the patient sample-reach $(n=1370)$

\begin{tabular}{lll}
\hline & Mean & SD \\
\hline Age $(n=1370)$ & 47.9 & 14.7 \\
Cigarettes per day $(n=1364)$ & 15.4 & 12.0 \\
& N & $\%$
\end{tabular}

Use of other tobacco in past 30 days $(n=1368)$

No
Yes
AUDIT-C (problem drinking) ( $n=1359$ )
$<4$ for males; $<3$ for females
$\geq 4$ for males; $\geq 3$ for females
PHQ2 (probable depression) $(n=1358$
PHQ $<3$
PHQ $\geq 3$
Psychiatric comorbidities $(n=1370)$
Primary psychotic
Bipolar
Unipolar
PTSD
Substance abuse

Substance abuse

Discharge diagnosis from ICD-9 codes $(n=1354)$

Infectious and parasitic diseases

Neoplasms

Endocrine, nutritional and metabolic diseases and immunity disorders

Diseases of the blood and blood-forming organs

Mental disorders

Diseases of the nervous system and sense organs

Diseases of the circulatory system

Diseases of the respiratory system

Diseases of the digestive system

Diseases of the genitourinary system

Complications of pregnancy, childbirth, and

the puerperium

Diseases of the skin and subcutaneous tissue

Diseases of the musculoskeletal system and connective tissue

Congenital anomalies

Certain conditions originating in the perinatal period

Symptoms, signs, and ill-defined conditions

Injury and poisoning

Procedures

Comorbidities or secondary discharge diagnoses from ICD-9 codes $(n=1370)$

Infectious and parasitic diseases

Neoplasms
Table 1 Baseline characteristics of the patient sample-reach $(n=1370)$ (Continued)

\begin{tabular}{|c|c|c|}
\hline $\begin{array}{l}\text { Endocrine, nutritional and metabolic diseases } \\
\text { and immunity disorders }\end{array}$ & 825 & 60.2 \\
\hline Diseases of the blood and blood-forming organs & 315 & 23.0 \\
\hline Mental disorders & 691 & 50.4 \\
\hline Diseases of the nervous system and sense organs & 394 & 28.8 \\
\hline Diseases of the circulatory system & 730 & 53.5 \\
\hline Diseases of the respiratory system & 470 & 34.3 \\
\hline Diseases of the digestive system & 486 & 35.5 \\
\hline Diseases of the genitourinary system & 296 & 21.6 \\
\hline $\begin{array}{l}\text { Complications of pregnancy, childbirth, and } \\
\text { the puerperium }\end{array}$ & 75 & 5.5 \\
\hline Diseases of the skin and subcutaneous tissue & 113 & 8.2 \\
\hline $\begin{array}{l}\text { Diseases of the musculoskeletal system and } \\
\text { connective tissue }\end{array}$ & 333 & 24.3 \\
\hline Congenital anomalies & 13 & 0.9 \\
\hline $\begin{array}{l}\text { Certain conditions originating in the perinatal } \\
\text { period }\end{array}$ & 0 & 0.0 \\
\hline Symptoms, signs, and ill-defined conditions & 485 & 35.4 \\
\hline Injury and poisoning & 254 & 18.5 \\
\hline \multicolumn{3}{|l|}{$\operatorname{Sex}(n=1370)$} \\
\hline Male & 667 & 48.7 \\
\hline Female & 703 & 51.3 \\
\hline \multicolumn{3}{|l|}{ Ethnicity $(n=1370)$} \\
\hline Non-Hispanic & 1323 & 96.6 \\
\hline Hispanic & 47 & 3.4 \\
\hline \multicolumn{3}{|l|}{ Race $(n=1370)$} \\
\hline White & 1051 & 76.7 \\
\hline African American/Black & 237 & 17.3 \\
\hline Other & 82 & 6.0 \\
\hline \multicolumn{3}{|l|}{ Education level $(n=1358)$} \\
\hline High school or lower & 765 & 56.3 \\
\hline Some college or higher & 593 & 43.7 \\
\hline \multicolumn{3}{|l|}{ Marital status $(n=1368)$} \\
\hline Married/domestic partner & 435 & 31.8 \\
\hline Separated/divorced/widowed & 490 & 35.8 \\
\hline Never married & 443 & 32.4 \\
\hline \multicolumn{3}{|l|}{ Employment status ( $n=1365)$} \\
\hline Employed & 393 & 28.8 \\
\hline Unemployed & 355 & 26.0 \\
\hline Retired/disabled/homemaker & 617 & 45.2 \\
\hline
\end{tabular}

(98.6\%), white (89.5\%), and only $4.9 \%$ reported smoking. About $68.3 \%$ had a 4-year degree and $97.6 \%$ were registered nurses (RNs). About $45.1 \%$ worked on medical surgical units, $21.7 \%$ worked on intensive care units (ICU/CCU/PCU), $11.6 \%$ worked on obstetric units, and 
$21.7 \%$ worked on other units (float, emergency, behavioral health, or outpatient/diagnostics).

\section{Participation in and results of nurse post-intervention surveys}

Nurse recruitment logs showed that across both intervention and control hospitals, $63.5 \%(n=1098 / 1730)$ of targeted nurses and 47 non-targeted providers returned 3-month follow-up surveys, for a total of 1145 participants. Post-intervention nurse survey response rates were $62.0 \%(n=802 / 1293)$ at intervention sites and $67.7 \%(n=296 / 437)$ at control sites. Nurse surveys showed that characteristics of the post-intervention sample of nurses was similar to the pre-intervention sample with one exception (educational differences) noted below. Note, the number of targeted nurses decreased pre- to post-intervention across intervention and control sites due to staff reductions (1829 pre- to 1730 postintervention). Additionally, $10.8 \%(n=140 / 1293)$ of nurses at intervention sites were interviewed.

\section{Participation in and opinions about the tobacco tactics training from nurse recruitment logs and surveys}

In the intervention hospitals only, recruitment logs showed that $76.0 \%(n=1028 / 1352)$ of targeted inpatient RN and licensed practical nurses (LPNs) and 317 additional, non-targeted providers employed at the time of intervention participated in the training, for a total of 1345 participants. Nearly all nurses who attended the training, $99.2 \% \quad(n=1334 / 1345)$, completed surveys immediately after training. About $92.4 \%$ were extremely/somewhat satisfied with the training, $97.9 \%$ rated pharmaceutical management as good/very good/ excellent, $97.3 \%$ rated behavioral management as good/ very good/excellent, $97.1 \%$ agreed or strongly agreed that they understood it, and $90.9 \%$ thought it was extremely/somewhat helpful.

\section{Implementation \\ Pre-to post-intervention changes in nurses' attitudes, behaviors, and barriers to implementing the intervention from surveys}

Table 2 shows that nurses in the intervention hospitals reported pre- to post-intervention increases in feeling that providing smoking cessation is important, feeling confident in providing smoking cessation services, and providing smoking cessation services, while there were significant decreases in the control hospitals. Nurses in the intervention hospitals reported increases in providing advice to quit, counseling, medications, handouts, and DVD, while there were no increases in the control hospitals. Moreover, nurses in the intervention hospitals reported significantly decreased barriers to implementing smoking cessation services, while there were marginally significant increases in selected barriers in the control hospitals. To determine reasons as to why the control hospitals actually did worse over time, post hoc analyses were conducted to determine if there were differences in the characteristics of the nurse sample pre- to postintervention. In the control hospitals only, $62.3 \%$ of nurses reported having at least a 4-year degree preintervention, but this dropped to $52.1 \%$ post-intervention $(p<0.05)$, while there was no significant change in the educational preparation of the nurses pre- to postintervention in the intervention group. On average, intervention site nurses spent about $8 \mathrm{~min}$ counseling each smoker.

\section{Implementation of services downloaded from the EMR}

To further determine nurses' charted provision of services in the intervention sites, post-intervention deidentified data was downloaded from the EMR documentation template from all smokers (enrolled in the study or not) during a 5-month period. As shown in Table 3, providing the brochure and Tobacco Tactics manual was charted in over $50 \%$ of identified smokers interested in quitting, while one in five participants was charted to have been given medications.

\section{Implementation of services from nurse interviews}

Of the 140 targeted nurses interviewed 2 to 6 months post-intervention at the intervention sites only, over $82.9 \%$ reported implementing all components of the intervention, except only $53.6 \%$ reported showing the DVD [44]. Qualitative comments from the nurse interviews shown in Table 4 indicate that nurses did not show the DVD largely because the overhead television system was not working or when it was working, it was cumbersome to use. Moreover, the nurses reported that charting was problematic as it took some time to integrate the documentation template into the EMR. Overall, the qualitative comments were very positive using words such as "user friendly," "streamlined," or "saves time" to describe the program and materials.

\section{Implementation of follow-up calls from volunteer telephone logs}

At the 3 intervention sites, volunteers made at least 1057 attempts/phone calls to 228 patients over a 5month period in 2013, of which $63.2 \%$ were reached at least once. An average of 2.2 follow-up phone calls per patient were completed to 144 patients, for a total of 313 patient contacts. The remaining attempts/phone calls resulted in voicemail messages, no answers, speaking with someone other than the patient, and unreachable phone numbers. 
Table 2 Changes in nurses' self-reported attitudes and behaviors regarding providing cessation services—implementation

\begin{tabular}{|c|c|c|c|}
\hline & Pre-intervention $=1345$ & Post-intervention $=849$ & Chi-square \\
\hline & Control = 375 & Control = 296 & \\
\hline & $n(\%)$ & $n(\%)$ & $P$ value \\
\hline Smoking cessatior & nportant & & \\
\hline Intervention & $1015(75.9)$ & $567(83.6)$ & $<0.001$ \\
\hline Control & $249(75.0)$ & $145(54.9)$ & $<0.001$ \\
\hline Very or extremely & orovide smoking cessatio & & \\
\hline Intervention & $382(28.6)$ & $387(57.1)$ & $<0.001$ \\
\hline Control & $141(43.1)$ & $80(30.3)$ & $<0.001$ \\
\hline Currently provide & ices & & \\
\hline Intervention & $1134(84.9)$ & $635(92.4)$ & $<0.001$ \\
\hline Control & $273(82.0)$ & $195(73.0)$ & 0.009 \\
\hline Smoking cessation & & & \\
\hline Advice & & & \\
\hline Intervention & $940(83.9)$ & $588(93.5)$ & $<0.001$ \\
\hline Control & $242(90.3)$ & $161(83.9)$ & 0.039 \\
\hline Individual coun & & & \\
\hline Intervention & $267(23.8)$ & $255(40.7)$ & $<0.001$ \\
\hline Control & $91(34.1)$ & $53(27.7)$ & 0.150 \\
\hline Group counseli & & & \\
\hline Intervention & $48(4.3)$ & $43(6.9)$ & 0.019 \\
\hline Control & $21(7.9)$ & $24(12.6)$ & 0.095 \\
\hline Medications & & & \\
\hline Intervention & $845(75.7)$ & $533(85.6)$ & $<0.001$ \\
\hline Control & $227(84.7)$ & $157(81.8)$ & 0.404 \\
\hline Handouts & & & \\
\hline Intervention & $1022(91.1)$ & $593(94.3)$ & 0.017 \\
\hline Control & $200(74.6)$ & $148(77.1)$ & 0.545 \\
\hline DVD & & & \\
\hline Intervention & $94(8.4)$ & $88(14.0)$ & $<0.001$ \\
\hline Control & $16(5.9)$ & $13(6.8)$ & 0.709 \\
\hline Phone calls & & & \\
\hline Intervention & $35(3.2)$ & $43(6.9)$ & $<0.001$ \\
\hline Control & $9(3.4)$ & $7(3.7)$ & 0.866 \\
\hline Face barriers that & ide smoking cessation se & & \\
\hline Intervention & $1042(78.9)$ & $431(64.2)$ & $<0.001$ \\
\hline Control & 199 (59.9) & $148(56.1)$ & 0.340 \\
\hline Barriers indicated: & & & \\
\hline Lack of confide & & & \\
\hline Intervention & $198(14.7)$ & $74(8.7)$ & $<0.001$ \\
\hline Control & $24(6.4)$ & $16(5.4)$ & 0.589 \\
\hline Not enough tra & & & \\
\hline Intervention & $435(32.3)$ & $42(4.9)$ & $<0.001$ \\
\hline Control & 78 (20.8) & $67(22.6)$ & 0.566 \\
\hline
\end{tabular}

Not enough time 
Table 2 Changes in nurses' self-reported attitudes and behaviors regarding providing cessation services—implementation (Continued)

\begin{tabular}{llll}
\hline Intervention & $665(49.4)$ & $246(29.0)$ & $<0.001$ \\
Control & $104(27.7)$ & $87(29.4)$ & 0.636 \\
Hesitant to upset patients & & $134(15.8)$ & $<0.001$ \\
Intervention & $378(28.1)$ & $53(17.9)$ & 0.083 \\
Control & $49(13.1)$ & $8(0.9)$ & $<0.001$ \\
Not my job & & $22(7.4)$ & 0.053 \\
Intervention & $50(3.7)$ & & \\
Control & $15(4.0)$ & & \\
\hline
\end{tabular}

Nurses from non-targeted units were intentionally not surveyed in the post-intervention period, thus the sample size is smaller than the pre-intervention period

\section{Maintenance/sustainability}

To enhance sustainability, training was incorporated into new hire nurse training at all sites. The information in Tables 2, 3, and 4, which was collected during the postintervention period, suggests that there was short-term sustainability. While data were not systematically collected on long-term sustainability of the program, there is anecdotal evidence that the program is being maintained, as members of the study team are periodically contacted by Trinity Health nurses with questions, primarily regarding where to order more materials. One of the intervention hospitals reported that they changed the nurse training from face-to-face to online training. Two other Trinity Health hospitals heard about the program, contacted us, and are implementing components of the intervention.

\section{Discussion}

Reach

The intervention had high reach as demonstrated by postintervention patients in the intervention sites reporting receiving significantly more handout materials. Unlike telephone quit lines, which have been shown to be highly effective, but reach only 6 to $10 \%$ of smokers [45], numerous studies have shown that inpatient cessation programs, including those delivered by nurses $[2,46]$, have the potential to reach a large number of captive smokers [47-50]. Participants in the sample had many characteristics that placed them at high risk for smoking and relapse back to smoking [51] including probable problem drinking, depression, and less than a high school education [52]. Diseases of the respiratory and circulatory systems were among the most common and are often smoking-related $[53,54]$, which may increase motivation to quit [55].

\section{Adoption}

One of our best measures of success is that over three quarters of targeted nurses participated in the Tobacco Tactics training on targeted units with additional participation from non-targeted units. The high satisfaction with the training was likely discussed among nurses and their managers resulting in many nurses attending from non-targeted units. The extension of the intervention to various types of intensive care units, outpatient, emergency room, psychiatric, substance abuse, and obstetric units (including expectant fathers) speaks not only to the to the quality of the program but also for the need for training providers to conduct tobacco cessation interventions in hospital settings.

Table 3 Nurses charted documentation on EMR template $(n=1388)$ _Implementation

\begin{tabular}{llll}
\hline Intervention type & $n$ & Percentage of total sample & Percentage of omitting refusers \\
\hline Refused intervention, given brochure & 251 & 18.1 & - \\
Accepted intervention, given brochure & 768 & 55.3 & 67.5 \\
Provided Tobacco Tactics manual & 578 & 41.6 & 50.8 \\
Provided nicotine replacement therapy & 252 & 18.2 & 22.1 \\
Provided behavioral counseling & 243 & 17.5 & 21.4 \\
Provided 1-800-QUIT-NOW card & 214 & 15.4 & 18.8 \\
Provided FDA smoking medication counseling & 213 & 15.3 & 18.7 \\
Shown DVD & 22 & 1.6 & 1.9 \\
Provided Bupropion or Chantix & 14 & 1.0 & 1.2 \\
\hline
\end{tabular}

$n=1388$ includes de-identified data from all smokers including those enrolled and not enrolled in the study during 5-month time period 
Table 4 Qualitative information provided during interviews) - Implementation

\begin{tabular}{ll}
\hline Component & Comments \\
\hline Brochure & Put brochure in patient's admission package \\
"I handed the brochure to the partner of a patient." & "I don't always provide the brochure. It is sometimes inconvenient." \\
DVD & "Aatients are usually not interested \\
"Accessing the DVD is too difficult for patients" & "I really like it." (5) \\
"User friendly." & "Materials make it easy; it's simple enough for patients with less education." \\
"The book saves me a lot of time talking." & "The book is informative and simple, that is good." \\
"The manual is kind of long and wordy." & "I have to admit that I had an attitude when I went to the training, but it was \\
"much more interesting than I thought." & "I liked the training. I feel more comfortable talking to patients now" \\
"The training gave me more ideas." & "I complement the program and planning. It is presented well and attainable." \\
"The training made me more comfortable talking to patients." \\
"I like that I can take this information and apply it to other issues as well." \\
"Training was mandatory on our unit." \\
"I did not attend the training, but learned the content from other nurses who went." \\
"I was too busy at the time to attend training." (2)
\end{tabular}

Tobacco Tactics intervention

Charting

What nurses say to patients

"This [Tobacco Tactics intervention] is more than we had before."

"The intervention is more streamlined now."

"This is a good service, the patients appreciate it; it's just that we're too busy with other things, so we don't always remember to address smoking."

"I give materials to fathers too." (nurse on OB/GYN unit)

"Charting is a problem" (11)

"Quitting will improve wound healing and overall health." (4)

"I say you haven't smoked this long, it's a good time to quit."

"The [quit] day is here."

"Never quit quitting!"

"Smoking is the \#1 modifiable risk factor."

"I focus on support group and home."

"Don't smoke in front of children."

Asks if spouse smokes.

"I encourage patients to write down how much money they safe each time they don't smoke for a day."

"I say: 'Either it is gas money, or it is cigarette money."

"I tell them: 'direct your brain to s.th. [something] else."

From an email: ... "just a few short hours after I was in your tobacco tactics class on Monday... I was taking care of a patient who smoked a pack a day and had wanted to quit for a long time. I sat down with her and told her how important it was for her health to quit smoking (yes, I used the phrase)! She agreed and said that she really wanted to quit but that she wasn't ready because she needed help. I reminded her that she wasn't going to be smoking while she was here (we had her on a nicotine patch) and that now would be a great time to quit. She agreed and said how she has her son's wedding coming up in December and she would love to be smoke-free by then. I explained how that would be an excellent goal and that she might as well start now! She looked at me, smiled, and said, "let's do it!" I gave her the Tobacco Tactics book and the 1-800-quit now card and she spent the rest of the evening looking through the book! She told the day shift RN during bedside report that I had convinced her to quit smoking and that she was going to stick with it! YAY!"

Cessation help strategies

"I provide counseling only if patients want to."

"It is important to keep reminding patients when they are in the hospital, since it is important for their health."

"Education is the biggest thing; I point out the benefits and provide examples from the patient's life, e.g.. I relate quitting to the grandchildren."

"Almost always gives support, but not always the brochure, will now."

"A patient with lumbar fusion will be offered Bupropion or Chantix."

"We should frame it in the baby's health framework."

"Removing cigarettes from home and work is a good implementation strategy for the patient."

Opinions on smoking and quitting

"Patients don't mind to hear about quitting from me." 
Table 4 Qualitative information provided during interviews) - Implementation (Continued)

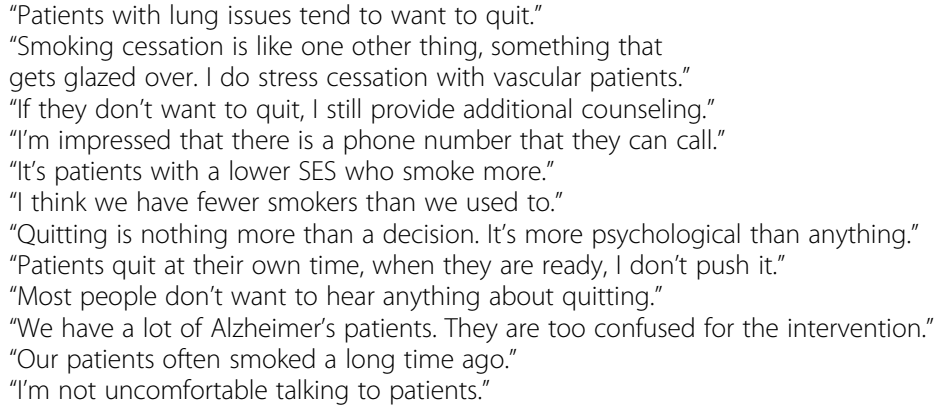

Nurses increased perceived importance of and selfconfidence in delivering tobacco cessation services likely contributed to the increased adoption of the intervention and subsequent increased quit rates in the intervention sites. Our prior work has shown that nurses who were more satisfied with and had a better understanding of the Tobacco Tactics intervention had significantly higher perceived confidence scores and importance scores related to providing the intervention [56]. Providers who feel more confident in their ability to do what is expected, recognize the need and importance for the intervention, and have the requisite skills are more likely to implement a program at higher levels of fidelity and result in increased quit rates [57-59].

\section{Implementation}

With as little as a 1-h training session, the proportion of nurses self-reporting the provision of cessation services significantly increased from pre- to post-intervention. This finding is congruent with a Cochrane Collaborative Review that demonstrated that health professional training increases the delivery of smoking cessation interventions [59]. Implementing standard protocols, which have been shown to be effective in increasing smoking cessation counseling interventions provided by nurses [60], likely enhanced service delivery [61]. Nurses are ideally positioned to deliver cessation interventions because (1) physician time is at a premium, (2) nurses are educated in psychosocial and physiological interventions, (3) nurses have access to and immediate rapport with patients as well as respect from physicians, (4) nurses understand the patient's medical condition and can tailor the intervention accordingly, and (5) nurses can read charts, initiate medication orders, and write nursing notes.

It is interesting to note that in the control group, nurses pre- to post-intervention self-reported a decrease in feeling that providing smoking cessation is important, a decrease in feeling confident in providing smoking cessation services, and less provision of smoking cessation services, and selected barriers increased at marginally significant levels. Moreover, patients in the control group reported receiving less smoking cessation handouts post-intervention compared to pre-intervention. This may be related to the lower educational levels of the nurses in the post-intervention period compared to the pre-intervention period. This is similar to another smoking cessation implementation study that also trained nurses and found that low performing units had nurses that were less educationally prepared [50].

Lack of time and competing demands have been cited as barriers to providing cessation services by nurses in other studies [62]. Yet, our work and the work of others $[63,64]$ have shown that the major barrier to nurses providing cessation services is not lack of time but lack of expertise, as the nurses in our current study reported that the intervention "saves time." Although in our prior VA study [20], splicing the DVD into the overhead television system was easy and nurses reported that showing the patient the DVD saved them time at the bedside, the DVD was not easily integrated into the overhead television system used by Trinity Health.

While several studies $[65,66]$ and this study report the use of the EMR to identify smokers, there are few studies [19] that use the EMR to actually document the treatment of smoking in accordance with JC standards [38]. While qualitative comments supported the need for improvement in the documentation template, there is motivation for hospital leadership to conduct this programming as meaningful use (the set of standards defined by the Centers for Medicare \& Medicaid Services Incentive Programs that governs the use of EMRs) will allow eligible providers and hospitals to earn incentive payments for implementing tobacco cessation strategies [67]. Currently, the JC is starting to work with Mathematica Policy Research and the Center for Medicare and Medicaid Services to assemble the Tobacco Treatment Task Force, which will reengineer these measures as electronic clinical quality measures.

\section{Maintenance}

The nurse-delivered Tobacco Tactics program remained sustainable 1 year after the researchers withdrew from 
the settings and longer term sustainability has been anecdotally verified. Integrating the program into new nurse training has enhanced sustainability as nurses turn over. While volunteers made phone calls during the study, unfortunately, there was no one from any of the intervention hospitals invested in coordinating the follow-up phone calls when the study ended. This is similar to our prior VA study that implemented and evaluated volunteer follow-up phone calls for smoking cessation and found it to be effective and cost-effective yet was not sustainable for similar reasons [68].

\section{Application of the RE-AIM framework to assess intervention implementation}

This study has shown how the RE-AIM framework can be used to guide research-based interventions in clinical practice. Utilization of the RE-AIM framework can serve as a guide to plan, conduct, and report on interventions that are implemented on a large scale in real-world settings [16]. Not only can the RE-AIM framework identify individual impact, but it can also identify population impact, as was done in this study [69]. The framework can be used to maximize external validity; report elements of both internal and external validity; review a body of evidence; and compare interventions to make policy decisions [23, 24, 70-73].

\section{Strengths and limitations of the study}

While pragmatic designs are more feasible and allow for implementation in natural environments [74], the lack of randomization may pose challenges in terms of internal validity, making it harder to rule out confounding variables [75]. Although data from one of the control hospitals did not materialize, the nurse and patient sample sizes in the other two control hospitals were large enough to allow clinically and statistically meaningful comparisons with the intervention hospitals. Nurses' implementation of the Tobacco Tactics intervention was based on self-report and therefore may have been inflated, although there was no inflation in the nurse self-report in the control group. Moreover, self-reported implementation of the Tobacco Tactics intervention was somewhat substantiated by EMR-downloaded documentation of services and qualitative comments by nurses reflected a large amount of detail about and enthusiasm for implementing the intervention.

\section{Conclusions}

The Tobacco Tactics intervention had high reach among inpatient smokers. Adoption and implementation were also high probably due to the packaging of the intervention into a user-friendly toolkit. Short-term maintenance was substantiated by patient and nurse surveys, and anecdotal evidence suggests that the program remains sustainable. Imagine the enormous reach and public health impact of an inpatient smoking cessation intervention if the largest group of front-line providers, namely nurses, were trained to effectively provide the tobacco cessation interventions. As we move toward more population-based interventions, the RE-AIM framework is a valuable guide for implementation.

\section{Additional files}

Additional file 1: Summary of RE-AIM measures. (DOCX $22 \mathrm{~kb}$ )

Additional file 2: Staff-3-month post-training survey. (DOCX 79 kb)

Additional file 3: Nurse interview guide. (DOCX $17 \mathrm{~kb}$ )

\section{Acknowledgements}

This work is greatly enhanced by the invaluable expertise of the personnel from all of the CHART research sites as well as William Riley, PhD, Catherine (Kate) Stoney, PhD, and other NIH personnel. The authors would like to express our heartfelt appreciation to the nurses and other staff who included the intervention in their already busy work schedules. The study could not have been accomplished without the hard work of our research staff and staff from the Trinity Health System, including Lucinda Wilkinson, Rebecca Masters, Rita Ferguson, Sonya Hilbrand, Danielle Poliski, John Ryan Scott, Julia Ferry, Elizabeth Tyrpak, Jennessa Rooker, Cody Carey, Tina Maxbauer, Seung Hee Choi, and Samantha Louzon. Lastly, we would like to thank the patients who participated in this study. A special acknowledgement goes to coauthor, David Ronis, who was the methodological mentor to Sonia Duffy as she developed the Tobacco Tactics intervention over the years and also provided statistical analyses for the vast majority of papers (including this one) related to the Tobacco Tactics intervention.

\section{Funding}

The project described was supported by Award Number U01HL105218 from the National Heart, Lung, and Blood Institute. The content is solely the responsibility of the authors and does not necessarily represent the official views of the National Heart, Lung, and Blood Institute or the National Institutes of Health (NIH).

\section{Availability of data and materials}

The datasets generated during and/or analyzed during the current study are not publicly available due to the large amount personal health information but are available from the corresponding author on reasonable request.

\section{Authors' contributions}

SD conceptualized the study, implemented the study, participated in analysis and created the manuscript. DR provided oversight of data collection and integrity and directed data analysis. LE served as the study project manager. AW served as the study intervention nurse. SH conducted data analysis. PT and GL provided oversight in the implementation of the study in the Trinity Health site. CW provided oversight in the implementation of the study in the St. Mary's Grand Rapids site. KM provided oversight in the implementation of the study at the Mercy Health Muskegon site. LF provided oversight in the implementation of the study at the St. Joseph Mercy Ann Arbor site. SK provided oversight in the implementation of the study at the St. Mary's Livonia site. NJ assisted with the writing of the paper. All authors read and approved the final manuscript.

\section{Competing interests}

The authors declare that they have no competing interests.

\section{Consent for publication}

Not applicable.

\section{Ethics approval and consent to participate}

Human studies approval was received from the University of Michigan (Health Sciences and Behavioral Sciences Institutional Review Board \#HUM00043349) and Trinity Health hospitals (Mercy Health Institutional Review Board \#2011013, 
Saint Joseph Mercy Institutional Review Board \#HSR-11-1272, and Saint Mary's Health Care Institutional Review board \#SM11-830-01).

\section{Author details}

${ }^{1}$ College of Nursing, Ohio State University, Newton Hall, 1585 Neil Ave, Columbus, OH 43210, USA. ${ }^{2}$ Veterans Affairs (VA) Center for Clinical Management Research, HSR\&D Center of Excellence, 2215 Fuller Road, Ann Arbor, MI 48105, USA. ${ }^{3}$ University of Michigan School of Nursing, 400 North Ingalls Building Room 4330, Ann Arbor, Ml 48109-0482, USA. ${ }^{4}$ VA Center for Clinical Management Research, HSR\&D Center of Excellence, 2215 Fuller Road, Ann Arbor, MI 48105, USA. Internal Medicine, Brehm Tower, University of Michigan, Room 6115, 1000 Wall Street, Ann Arbor, Ml 48109-5714, USA. ${ }^{6}$ Trinity Health, 20555 Victor Parkway, Livonia, Ml 48152, USA. ${ }^{7}$ The Lacks Cancer Center, Mercy Health Saint Mary's, 200 Jefferson SE, Grand Rapids, MI 49503, USA. ${ }^{8}$ Mercy Health Partners, 1500 Sherman Blvd., Muskegon, MI 49444, USA. ${ }^{9}$ Saint Joseph Mercy Health System, 5305 E. Huron River Dr., Ann Arbor, Ml 48106-0995, USA. ${ }^{10}$ Saint Mary Mercy Hospital, 36475 Five Mile Road, Livonia, Ml 48154-1988, USA. "'Department of Psychiatry and Behavioral Sciences, Northwestern University, Feinberg School of Medicine, Abbott Hall 710 North Lake Shore Drive, Suite 904, Chicago, IL 60611, USA.

${ }^{12}$ Center for Management of Complex Chronic Care, Hines VA Hospital, 5000 S 5th Ave., Hines, IL 60141, USA. ${ }^{13}$ Dartmouth-Hitchcock Medical Center, One Medical Center Dr., Lebanon, NH 03756, USA.

\section{Received: 29 March 2016 Accepted: 17 October 2016} Published online: 04 November 2016

\section{References}

1. Rigotti NA, et al. Interventions for smoking cessation in hospitalised patients. Cochrane Database Syst Rev. 2012;5(5):CD001837.

2. Rice VH, Hartmann-Boyce J, Stead LF. Nursing interventions for smoking cessation. Cochrane Database Syst Rev. 2013;8.

3. Simon JA, et al. Smoking cessation after surgery. A randomized trial. Arch Intern Med. 1997;157(12):1371-6.

4. Simon JA, et al. Intensive smoking cessation counseling versus minimal counseling among hospitalized smokers treated with transdermal nicotine replacement: a randomized trial. Am J Med. 2003;114(7):555-62.

5. Taylor CB, Curry SJ. Implementation of evidence-based tobacco use cessation guidelines in managed care organizations. Ann Behav Med. 2004;27(1):13-21.

6. France EK, Glasgow RE, Marcus AC. Smoking cessation interventions among hospitalized patients: what have we learned? Prev Med. 2001;32(4):376-88.

7. Reid $J$, et al. Socioeconomic disparities in quit intentions, quit attempts, and smoking abstinence among smokers in four western countries: findings from the International Tobacco Control Four Country Survey. Nicotine Tob Res. 2010;12(Suppl):S20-33.

8. Reid RD, et al. Smoking cessation for hospitalized smokers: an evaluation of the "Ottawa Model". Nicotine Tob Res. 2010;12(1):11-8.

9. Prochaska JJ, et al. Designing for dissemination: development of an evidence-based tobacco treatment curriculum for psychiatry training programs. J Am Psychiatr Nurses Assoc. 2009;15(1):24-31.

10. Anesetti-Rothermel $A$, et al. Beyond reach and effectiveness: evaluating the not-on-tobacco (N-o-T) program in West Virginia from 2000 to 2005. Health Promot Pract. 2011;13(4):506-14.

11. Meyer $\mathrm{C}$, et al. Adoption, reach and effectiveness of computer-based, practitioner delivered and combined smoking interventions in general medical practices: a three-arm cluster randomized trial. Drug Alcohol Depend. 2011;121(1-2):124-32.

12. Caperchione C, Coulson F. The WellingTONNE Challenge Toolkit: using the RE-AIM framework to evaluate a community resource promoting healthy lifestyle behaviours. Health Educ J. 2010;69(1):126-34.

13. Abildso CG, Zizzi SJ, Reger-Nash B. Evaluating an insurance-sponsored weight management program with the RE-AIM model, West Virginia, 20042008. Prev Chronic Dis. 2010;7(3):A46.

14. Gaglio B, Shoup JA, Glasgow RE. The RE-AIM framework: a systematic review of use over time. Am J Public Health. 2013;103(6):e38-46.

15. Kessler RS, et al. What does it mean to "employ" the RE-AIM model? Eval Health Prof. 2013;36(1):44-66.

16. Virginia Polytechnic Institute and State University. RE-AIM. 2013; Available from: http://www.re-aim.org/. Accessed 13 Sept 2013.
17. Bakken S, Ruland CM. Translating clinical informatics interventions into routine clinical care: how can the RE-AIM framework help? J Am Med Inform Assoc. 2009;16(6):889-97.

18. Duffy SA, et al. A tailored smoking, alcohol, and depression intervention for head and neck cancer patients. Cancer Epidemiol Biomarkers Prev. 2006;15(11):2203-8.

19. Duffy SA, et al. Implementation of the Tobacco Tactics program in the Department of Veterans Affairs. J Gen Intern Med. 2010;25 Suppl 1:3-10.

20. Duffy SA, et al. Effectiveness of the Tobacco Tactics program in the Department of Veterans Affairs. Ann Behav Med. 2014;48(2):265-74.

21. Vick $L$, et al. Implementation of an inpatient smoking cessation programme in a Veterans Affairs facility. J Clin Nurs. 2013; 22(5-6):866-80.

22. Choi SH, et al. Web-enhanced tobacco tactics with telephone support versus 1-800-QUIT-NOW telephone line intervention for operating engineers: randomized controlled trial. J Med Internet Res. 2014;16(11):e255.

23. Glasgow RE, et al. The future of health behavior change research: what is needed to improve translation of research into health promotion practice? Ann Behav Med. 2004;27(1):3-12.

24. Glasgow RE, Vogt TM, Boles SM. Evaluating the public health impact of health promotion interventions: the RE-AIM framework. Am J Public Health. 1999:89(9):1322-7.

25. Duffy SA, et al. Dissemination of the nurse-administered Tobacco Tactics intervention versus usual care in six Trinity community hospitals: study protocol for a comparative effectiveness trial. Trials. 2012;13(1):125.

26. Cummins $S$, et al. Nicotine patches and quitline counseling to help hospitalized smokers stay quit: study protocol for a randomized controlled trial. Trials. 2012;13(128):128.

27. Fellows $\mathrm{J}$, et al. Health and economic effects from linking bedside and outpatient tobacco cessation services for hospitalized smokers in two large hospitals: study protocol for a randomized controlled trial. Trials. 2012; 13(129):129

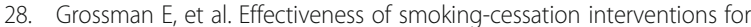
urban hospital patients: study protocol for a randomized controlled trial. Trials. 2012:13(126):126

29. Harrington $\mathrm{KF}$, et al., Web-based smoking cessation intervention that transitions from inpatient to outpatient: study protocol for a randomized controlled trial. Trials. 2012;13:123.

30. Reid ZZ, et al. Comparative effectiveness of post-discharge strategies for hospitalized smokers: study protocol for the Helping HAND 2 randomized controlled trial. BMC Public Health. 2015;15(109):1484.

31. Japuntich SJ, et al. Comparative effectiveness of post-discharge interventions for hospitalized smokers: study protocol for a randomized controlled trial. Trials. 2012;13:124.

32. Richter KP, Faseru B, Mussulman LM, Ellerbeck EF, Shireman TI, Hunt JJ, Carlini BH, Preacher KJ, Ayars CL, Cook DJ. Using "warm handoffs" to link hospitalized smokers with tobacco treatment after discharge: study protocol of a randomized controlled trial. Trials, 2012. 13(127)

33. Riley WT, et al. Overview of the Consortium of Hospitals Advancing Research on Tobacco (CHART). Trials. 2012:13(122):122.

34. Duffy SA, et al. Effectiveness of the Tobacco Tactics Program in the Trinity Health System. Am J Prev Med. 2016:51(4):551-65.

35. Glasgow RE, et al. National Institutes of Health approaches to dissemination and implementation science: current and future directions. Am J Public Health. 2012;102(7):1274-81.

36. Dillman DA. Mail and telephone surveys: the total design method. New York: John Wiley \& Sons; 1978.

37. Cooke F, et al. Diagnostic accuracy of NicAlert cotinine test strips in saliva for verifying smoking status. Nicotine Tob Res. 2008;10(4):607-12.

38. Joint commission on accreditation of healthcare organizations. Tobacco Treatment Measures (TTM). 2011; Available from: http://www. jointcommission.org/assets/1/6/Tobacco\%20Treatment\%20Measures\%20 List1.PDF. Accessed 2 Feb 2012.

39. Ewing LA, et al.. Development of the Tobacco Tactics logo: from thumb prints to press. Tob Induc Dis. 2012;10(1):6.

40. Endres C. Diseases and Injuries Tabular Index. 2011 June 20, 2014; Available from: http://icd9.chrisendres.com/index.php?action=contents.. Accessed 15 Apr 2016.

41. Lowe B, Kroenke K, Grafe K. Detecting and monitoring depression with a two-item questionnaire (PHQ-2). J Psychosom Res. 2005;58(2):163-71.

42. Bush $\mathrm{K}$, et al. The AUDIT alcohol consumption questions (AUDIT-C): an effective brief screening test for problem drinking. Ambulatory Care Quality 
Improvement Project (ACQUIP). Alcohol Use Disorders Identification Test. Arch Intern Med. 1998;158(16):1789-95.

43. Dawson DA, et al. Effectiveness of the derived Alcohol Use Disorders Identification Test (AUDIT-C) in screening for alcohol use disorders and risk drinking in the US general population. Alcohol Clin Exp Res. 2005;29(5):844-54.

44. Duffy SA, et al. Fidelity monitoring across the seven studies in the Consortium of Hospitals Advancing Research on Tobacco (CHART). Tob Induc Dis. 2015;13(1):29.

45. Tzelepis F, et al. Increasing the reach of quitlines through active telephone recruitment: do cold-called smokers differ from quitline callers? Nicotine Tob Res. 2012;14(12):1488-93.

46. DeBusk RF, et al. A case-management system for coronary risk factor modification after acute myocardial infarction. Ann Intern Med. 1994;120(9):721-9.

47. Duffy SA, et al. In-hospital smoking cessation programs: what do VA patients and staff want and need? Appl Nurs Res. 2008:21(4):199-206.

48. Hennrikus DJ, et al. The TEAM project: the effectiveness of smoking cessation intervention with hospital patients. Prev Med. 2005:40(3):249-58.

49. Wolfenden $\mathrm{L}$, et al. Helping hospital patients quit: what the evidence supports and what guidelines recommend. Prev Med. 2008;46(4):346-57.

50. Katz DA, et al. Implementing best evidence in smoking cessation treatment for hospitalized veterans: results from the VA-BEST trial. Jt Comm J Qual Patient Saf. 2014;40(11):493-1.

51. Duffy SA, et al. Risk of smoking and receipt of cessation services among veterans with mental disorders. Psychiatr Serv. 2012;63(4):325-32.

52. Hiscock $\mathrm{R}$, et al. Socioeconomic status and smoking: a review. Addiction Reviews. 2012;1248:107-23.

53. Centers for Disease, C. and Prevention. Annual smoking-attributable mortality, years of potential life lost, and productivity losses-United States, 1997-2001. MMWR Morb Mortal Wkly Rep. 2005;54(25):625-8.

54. Mohiuddin SM, et al. Intensive smoking cessation intervention reduces mortality in high-risk smokers with cardiovascular disease. Chest. 2007; 131(2):446-52.

55. Duffy SA, et al. Medical comorbidities increase motivation to quit smoking among veterans being treated by a psychiatric facility. Perspectives in Psychiatric Care. 2011;47(2):74-83.

56. Fore AM, et al. Nurses' delivery of the Tobacco Tactics intervention at a Veterans Affairs Medical Center. J Clin Nurs. 2014;23(15-16):2162-9.

57. Durlak JA, DuPre EP. Implementation matters: a review of research on the influence of implementation on program outcomes and the factors affecting implementation. Am J Community Psychol. 2008:41(3-4):327-50.

58. Fiore MC, et al. Treating tobacco use and dependence: 2008 update, Clinical Practice Guideline. Rockville: U.S. Department of Health and Human Services. Public Health Service; 2008.

59. Carson KV, et al. Training health professionals in smoking cessation. Cochrane Database Syst Rev. 2012;5:CD000214.

60. Nolan E, et al. Rapid-cycle improvement in quality of care for patients hospitalized with acute myocardial infarction or heart failure: moving from a culture of missed opportunity to a system of accountability. J Cardiovasc Manag. 2005;16(1):14-9.

61. Katz DA, et al. Implementing smoking cessation guidelines for hospitalized veterans: effects on nurse attitudes and performance. J Gen Intern Med. 2013;28(11):1420-9.

62. McCarty MC, et al. Nurses' attitudes concerning the delivery of brief cessation advice to hospitalized smokers. Prev Med. 2001;33(6):674-81.

63. Willaing I, Ladelund S. Smoking behavior among hospital staff still influences attitudes and counseling on smoking. Nicotine Tob Res. 2004;6(2):369-75.

64. Braun BL, et al. Smoking-related attitudes and clinical practices of medical personnel in Minnesota. Am J Prev Med. 2004;27(4):316-22.

65. Fraser $\mathrm{D}$, et al. Electronic health records as a tool for recruitment of participants' clinical effectiveness research: lessons learned from tobacco cessation. Transl Behav Med. 2013;3(3):244-52.

66. Piper ME, et al. Recruiting and engaging smokers in treatment in a primary care setting: developing a chronic care model implemented through a modified electronic health record. Transl Behav Med. 2013;3(3):253-63.

67. HITEC-LA. Meaningful Use Stage 1 Requirements. 2010; Available from: http://www.hitecla.org/meaningful_use_requirements. Accessed 24 Sept 2013.

68. Duffy SA, et al. Evaluation and costs of volunteer telephone cessation follow-up counseling for Veteran smokers discharged from inpatient units: a quasi-experimental, mixed methods study. Tob Induc Dis. 2015;13(1):4.
69. Glasgow RE, et al. Evaluating the impact of health promotion programs: using the RE-AIM framework to form summary measures for decision making involving complex issues. Health Educ Res. 2006;21(5):688-94.

70. Glasgow RE, Lichtenstein E, Marcus AC. Why don't we see more translation of health promotion research to practice? Rethinking the efficacy-toeffectiveness transition. Am J Public Health. 2003;93(8):1261-7.

71. Estabrooks PA, Gyurcsik NC. Evaluating the public health impact of physical activity interventions. Psychology of Sports and Exercise. 2003;73:21-8.

72. Glasgow RE. Evaluation of theory-based interventions: the RE-AIM model. In: Glanz K, Lewis FM, Rimer BK, editors. Health behavior and health education. San Francisco: Wiley; 2002.

73. Lando HA, et al. Promoting smoking abstinence in pregnant and postpartum patients: a comparison of 2 approaches. Am J Manag Care. 2001;7(7):685-93.

74. Trochim WM. The research methods knowledge base. QuasiExperimental Design 2006 October 20, 2006 [cited 2015 April 1]; Available from: http://www.socialresearchmethods.net/kb/quasiexp.php.

75. Robson $\mathrm{LS}$, et al. Quasi-experimental and experimental designs: more powerful evaluation designs, in Guide to Evaluating the Effectiveness of Strategies for Preventing Work Injuries. Department of Health and Human Services: Cincinnati, OH; 2001, p. 29-42.

\section{Submit your next manuscript to BioMed Central and we will help you at every step:}

- We accept pre-submission inquiries

- Our selector tool helps you to find the most relevant journal

- We provide round the clock customer support

- Convenient online submission

- Thorough peer review

- Inclusion in PubMed and all major indexing services

- Maximum visibility for your research

Submit your manuscript at www.biomedcentral.com/submit
O) Biomed Central 